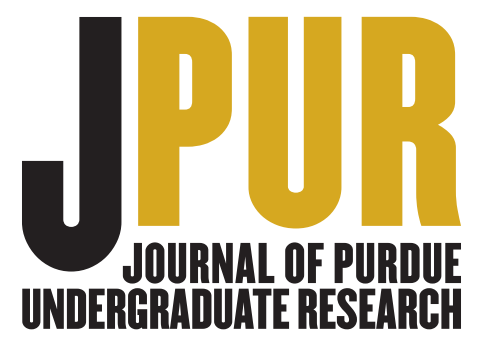

\title{
STEM
}

\section{The Impact of Bacteriophage \\ Therapy on Gut Microbiome}

\section{Student researcher: Kyle Schmidt, Freshman}

Phage therapy is the use of bacteriophages to treat bacterial infections. Unlike antibiotics, phages have a very narrow spectrum and often target single species or strains of bacteria. The effect of phage therapy on non-targeted bacteria, however, is largely unstudied. In this study, the impacts of orally administered phage therapy and antibiotic therapy on the composition of the gut microbiome, the complex bacterial ecosystem of the digestive system, were compared. Piglets $(n=17 ; 3$ weeks of age) were randomly assigned to one of three treatments: (1) ASP250 [antibiotic]; (2) Salmonella phage cocktail; and (3) control. All pigs were euthanized after 14 days of treatment. Samples were collected from cecum, ileum, and fecal contents.

A $16 \mathrm{~S}$ sequencing analysis of samples revealed that there were no differences in operational taxonomical unit (OTU) abundance and phylogeny in the gut microbiome of phage-treated and control pigs after 14 days of treatment.

OTU abundance in the microbiome of the ileum in antibiotictreated pigs differed significantly $(p<0.05)$ from that of both phage-treated and control pigs, and the ileum in antibiotictreated pigs had significantly higher percentages of clostridia and significantly lower percentages of Lactobacilli compared to the ileum in control pigs. Several OTUs representing genera including Clostridium, Prevotella, and Helicobacter were over- or underrepresented in pigs in both the antibiotic and phage groups when compared to the control pigs. Taken together, these data indicate that phage therapy may affect intestinal microbiomes. Determining whether these changes are detrimental will necessitate further study.
Research advisor Paul Ebner writes: "Phage therapy holds a lot of promise in preventing different types of bacterial infections, but there are still many unanswered questions. Kyle's work begins to assess the impact these treatments may have on the host. Specifically, he looked at whether phage treatments affect surrounding, potentially beneficial, bacteria."

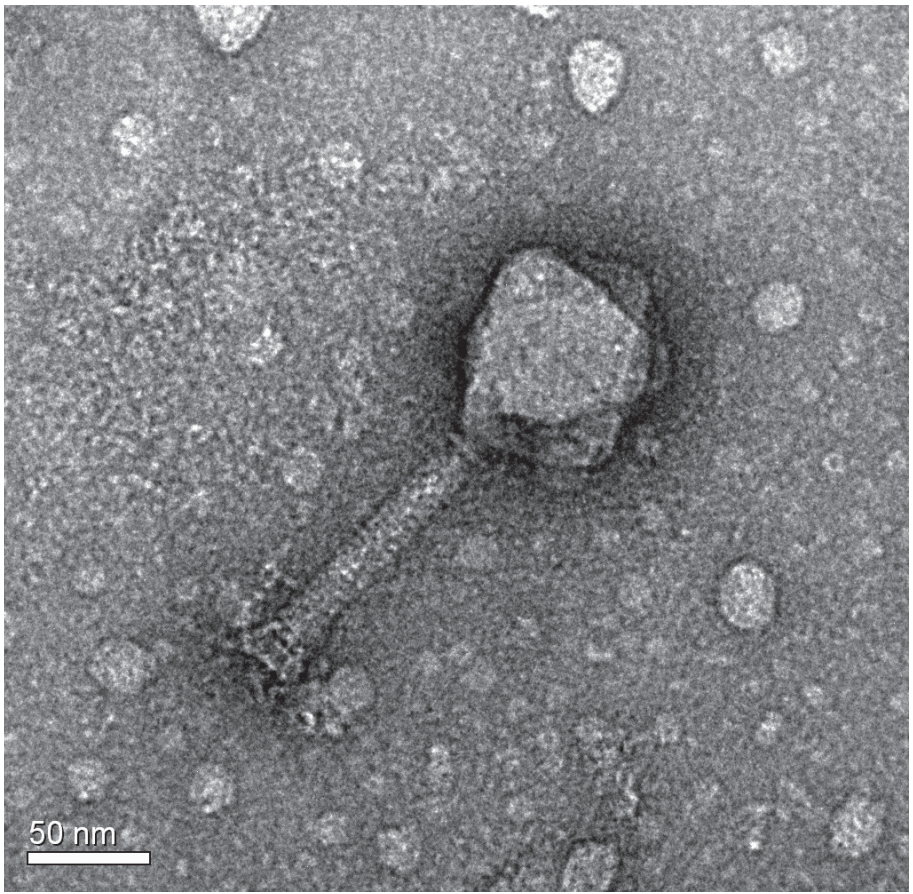

Electron micrograph of a bacteriophage. 